\title{
Maternal intravenous immunoglobulin: A non-invasive treatment option for Rhesus D-sensitised women with previous adverse
} pregnancy outcomes

\author{
N Frank, ${ }^{1}$ MB ChB, MMed, FCOG (SA); P Naidoo, ${ }^{1}$ MB ChB, MMed, FCOG (SA), Cert Maternal Fetal Med; \\ E Nicolaou, ${ }^{1,2,3} \mathrm{MD}$, FCOG (SA), Dip Fetal Med (UK) \\ ${ }^{1}$ Department of Obstetrics and Gynaecology, Chris Hani Baragwanath Hospital, University of the Witwatersrand, Johannesburg, South Africa \\ ${ }^{2}$ Morningside Mediclinic, University of the Witwatersrand, Johannesburg, South Africa \\ ${ }^{3}$ Department of Obstetrics and Gynecology, Pavilion for Women, Texas Children's Hospital, Baylor College of Medicine, Houston, USA
}

Corresponding author: N Frank (nadiya.frank@gmail.com)

\begin{abstract}
Background. Maternal intravenous immunoglobulin (IVIG) may delay the onset and severity of fetal anaemia in Rhesus D (RhD)sensitised pregnancies, thereby minimising the need for intrauterine transfusion and its associated complications.

Objective. To compare the pregnancy outcomes of RhD-sensitised women who received antenatal IVIG with those who did not receive antenatal IVIG.

Methods. This was a retrospective cross-sectional analysis of $\mathrm{RhD}$-sensitised women who attended the Wits Fetal Medicine Centre (Johannesburg) from 1 January 2008 to 31 May 2018. Criteria for maternal IVIG administration were: $(i)$ previous adverse pregnancy outcome (early neonatal death, intrauterine fetal death or miscarriage related to RhD sensitisation), (ii) women with high antibody titre levels $(\geq 1: 64)$ in the absence of fetal anaemia; and (iii) rising antibody titre levels. Maternal antibody titre levels, pregnancy and neonatal outcomes were compared in women who received IVIG v. those who did not receive IVIG.

Results. Of the $42 \mathrm{RhD}$-sensitised women, 14 received IVIG. A greater proportion of women experienced a decrease in antibody titres in the IVIG v. no-IVIG group ( $43 \%$ v. $11 \%$, respectively; $p=0.04$ ). Nine of the 10 women in the IVIG group with a previous adverse pregnancy outcome had a successful pregnancy outcome following IVIG treatment.

Conclusion. Maternal IVIG may provide a successful pregnancy outcome in RhD-sensitised women with previous adverse pregnancy outcomes related to Rh disease, or women with raised or increasing maternal antibody titre levels who present in the first or early second trimester.
\end{abstract}

S Afr J Obstet Gynaecol 2020;26(1):4-7. https://doi.org/10.7196/SAJOG.2020.v26i1.1514

Rhesus $\mathrm{D}(\mathrm{RhD})$ sensitisation refers to the development of maternal antibodies in response to foreign $\mathrm{RhD}$ antigens present on fetal red blood cells. Although rare, occurring in $1-2$ per 1000 pregnant women, there is a potential for devastating fetal and neonatal complications. ${ }^{[1]}$ Maternal antibodies that cross the placenta and destroy fetal red blood cells may result in fetal anaemia, hydrops or intrauterine death. Knowledge of fetal Rh status would assist in the management of $\mathrm{RhD}$-sensitised pregnancies by excluding $\mathrm{Rh}$ negative fetuses from further surveillance. High-throughput noninvasive prenatal testing (NIPT) is sufficiently accurate to detect fetal $\mathrm{RhD}$ status in $\mathrm{RhD}$-negative women, and would considerably reduce unnecessary treatment with routine anti-D immunoglobulin. The applicability of these findings to non-white women and women with multiple pregnancies is uncertain. ${ }^{[2]}$ However, this test is not currently available in South Africa (SA), where the current management of a $\mathrm{RhD}$-sensitised pregnancy involves monitoring maternal Rh antibody titre levels at 2 - 4-weekly intervals. Antibody titre levels are reported as a reciprocal of the last dilution that shows a positive agglutination reaction, as is the convention of laboratories in SA. RhD-sensitised women with an antibody titre level of $\geq 1: 16$ should be referred to a fetal medicine centre for further antibody titre monitoring and Doppler surveillance.
Antibody titre levels $\geq 1: 128$ may be associated with fetal anaemia. ${ }^{[3]}$ Technological advancements, with the use of Doppler studies, have led to a better understanding of fetal physiology and fetal response to anaemia. The fetus at risk for anaemia is screened non-invasively by measurement of the middle cerebral artery peak systolic velocity (MCA PSV) by Doppler ultrasound. If the MCA PSV is greater than 1.5 multiples of the median (MoM), fetal blood sampling is performed to confirm the diagnosis of fetal anaemia. ${ }^{[4]}$ The management of an anaemic fetus may include intrauterine transfusion (IUT) or delivery, and this in turn depends on gestational age, fetal weight, resources for neonatal care and maternal and fetal wellbeing. The decision to deliver is made by balancing the risk of repeated IUT against delivery of an anaemic or hydropic preterm neonate and postnatal transfusion or intravenous immunoglobulin (IVIG) administration thereafter. Despite the above advancements in the monitoring of Rh-sensitised pregnancies and the detection of fetal anaemia, the ideal treatment would prevent fetal anaemia, thereby minimising the need for IUT and its associated complications. The use of maternal hyperimmune globulin has been proposed to delay the onset and severity of fetal anaemia in Rh-sensitised pregnancies. ${ }^{[5]}$ Mechanisms of action of hyperimmune globulin include reducing maternal antibody 
production, inhibiting the passage of antibodies across the placenta and decreasing fetal phagocytosis of sensitised red blood cells through direct inhibition of the fetal reticuloendothelial system. ${ }^{[5]}$ Maternal IVIG may, therefore, offer a clinically beneficial alternative for the treatment of $\mathrm{RhD}$-sensitised women who are at high risk of developing fetal complications. There is limited evidence for the use of IVIG in the management of Rh-sensitised pregnancies. The aim of our study was to assess the pregnancy outcomes of RhDsensitised women who received antenatal IVIG against those who did not receive antenatal IVIG.

\section{Methods}

A retrospective cross-sectional analysis was performed for $\mathrm{RhD}$ sensitised women who attended the Wits Fetal Medicine Centre (WFMC; Morningside Mediclinic, Johannesburg) from 1 January 2008 to 31 May 2018. The WFMC attends to both high- and lowrisk women who are self-referred or referred by their attending gynaecologist. RhD-sensitised women were referred with an initial RhD titre.

\section{Maternal IVIG administration}

The criteria for first to early second trimester maternal IVIG administration in $\mathrm{RhD}$-sensitised women were: (i) previous adverse pregnancy outcome related to $\mathrm{RhD}$ sensitisation; (ii) high antibody titre levels $(\geq 1: 64)$ in the absence of fetal anaemia; or (iii) increasing antibody titre levels. Previous adverse pregnancy outcomes included early neonatal death (ENND), intrauterine fetal death (IUFD) and miscarriage related to $\mathrm{RhD}$ sensitisation. IVIG was administered at a dose of $0.5 \mathrm{~g} / \mathrm{kg}$ twice weekly until delivery. Women who were agreeable to treatment with IVIG were admitted to a short-stay medical ward for treatment under observation by their referring doctor. If there were no adverse effects, they were discharged later the same day or the following day. Referring doctors were asked to report any adverse treatment events and treatment thereof to the WFMC. Fetuses were assessed by ultrasound every 2 - 4 weeks and maternal antibody titre levels were taken every month.

\section{Screening and management of fetal anaemia}

Doppler ultrasound surveillance of the MCA for fetal anaemia was performed from 24 weeks' gestation. An MCA PSV >1.5 MoM was used to screen for fetal anaemia. Fetuses that had an MCA PSV $>1.5$ MoM were assessed for delivery or IUT. A single course of antenatal corticosteroids for fetal lung maturity was administered to all fetuses with suspected fetal anaemia that were $<34$ weeks' gestation regardless of the decision for IUT or delivery. Fetuses having an estimated fetal weight (EFW) of $>1.2 \mathrm{~kg}$ were delivered and transfused postdelivery as necessary. A cordocentesis was performed to confirm fetal anaemia when the EFW was $<1.2 \mathrm{~kg}$. IUT was performed at the time of cordocentesis if the $\mathrm{Hb}$ was $<10 \mathrm{~g} / \mathrm{dL}$ or if there was evidence of hydrops fetalis.

\section{Data collection}

The WFMC register was searched for all RhD-sensitised women who attended the centre from 1 January 2008 until 31 May 2018. The medical records of these women were reviewed, and the relevant details anonymously captured. All Rh-negative women with atypical antibodies, e.g. Kidd, Duffy, Kell, etc., were excluded. The following data were captured from medical records: demographic data, previous obstetric history, maternal antibody titre levels, fetal and maternal Doppler ultrasounds, IUT, IVIG administration, dosing and side-effects, delivery, maternal outcomes, fetal outcomes and neonatal outcomes. A descriptive analysis of fetal and pregnancy outcomes in Rh-sensitised women was performed. In addition, a comparative analysis of maternal antibody titre levels, pregnancy and neonatal outcomes was performed in women who did and did not receive IVIG. Data were analysed with the aid of a statistician using Stata version 14 (StataCorp., USA). Descriptive statistics were applied using medians with ranges and numbers with percentages. The $\chi^{2}$ test was used for comparison of frequencies, and Fisher's exact test was used where the sample number was $<5$. A $p$-value of $<0.05$ was used to define statistical significance. Ethics approval was obtained from the Human Research and Ethics Committee of the University of the Witwatersrand (ref. no. M181085).

\section{Results}

During the 10-year study period, $42 \mathrm{RhD}$-sensitised women attended the WFMC, and 14 of these received IVIG. The mean maternal age and parity of RhD-sensitised women was similar between those receiving and those not receiving IVIG (Table 1). The mean gestational age at administration of IVIG was 14 weeks, with a range of 19 - 30 weeks. There were no adverse maternal reactions to IVIG requiring additional investigations, treatment or hospital admission. Maternal antibody titre levels $>1: 128$ were observed in $64 \%$ of women in the IVIG group, compared with $0 \%$ of women in the no-IVIG group ( $p=0.01$; Table 1 ). Forty-three percent of women in the IVIG group had a decrease in maternal antibody titre levels, compared with $11 \%$ of women in the no-IVIG group $(p=0.04)$. For obstetric and neonatal outcomes by IVIG group, there was an equal frequency of fetuses that were born alive (92\%; Table 3). The median

Table 1. Antibody titre trends of RhD-sensitised women by treatment group

\begin{tabular}{llll}
\hline Category & $\begin{array}{l}\text { IVIG } \\
(\boldsymbol{n = 1 4}), \boldsymbol{n}(\mathbf{\%})\end{array}$ & $\begin{array}{l}\text { No-IVIG } \\
(\boldsymbol{n}=\mathbf{2 8}), \boldsymbol{n}(\mathbf{\%})\end{array}$ & $\boldsymbol{p}$-value \\
\hline Initial titre $\geq 1: 16$ & $14(100)$ & $11(39)$ & 0.01 \\
Initial titre $\geq 1: 128$ & $9(64)$ & 0 & 0.01 \\
Titre decrease & $6(43)$ & $3(11)$ & 0.04 \\
Titre increase & $2(14)$ & $5(18)$ & 0.57 \\
No titre change & $6(43)$ & $20(71)$ & 0.19 \\
RhD = Rhesus D; IVIG = intravenous immunoglobulin. & &
\end{tabular}

Table 2. Participant characteristics of $\mathrm{RhD}$-sensitised women by treatment group

\begin{tabular}{|c|c|c|c|}
\hline Characteristic & $\begin{array}{l}\text { IVIG } \\
(n=14), n(\%)^{*}\end{array}$ & $\begin{array}{l}\text { No-IVIG } \\
(n=28), n(\%)^{*}\end{array}$ & $p$-value \\
\hline $\begin{array}{l}\text { Age (years), median } \\
\text { (range) }\end{array}$ & $34(31-30)$ & $35(28-43)$ & 0.28 \\
\hline Parity, median (range) & $1(1-3)$ & $1(0-3)$ & 0.17 \\
\hline \multicolumn{4}{|l|}{ Previous adverse outcome } \\
\hline Total & $10(71)$ & $1(3.5)$ & \multirow[t]{3}{*}{$<0.01$} \\
\hline ENND & $8(57)$ & $1(3.5)$ & \\
\hline IUFD & $2(14)$ & 0 & \\
\hline $\begin{array}{l}\text { GA at delivery (weeks), } \\
\text { median (range) }\end{array}$ & $33(31-37)$ & $37(20-39)$ & $<0.01$ \\
\hline $\begin{array}{l}\mathrm{RhD}=\text { Rhesus } \mathrm{D} ; \mathrm{IVIG}=\text { intrav } \\
\text { IUFD = intrauterine fetal death, } \\
{ }^{*} \text { Unless otherwise specified. }\end{array}$ & $\begin{array}{l}\text { s immunoglobulin; } \mathrm{E} \\
=\text { gestational age. }\end{array}$ & $\mathrm{ND}=$ early neonata & \\
\hline
\end{tabular}


gestational age at delivery was 33 weeks in the IVIG group and 37 weeks in the no-IVIG group $(p=0.01)$. The median birth weight in the IVIG group was $2180 \mathrm{~g}$, compared with $2990 \mathrm{~g}$ in the no-IVIG group $(p=0.01)$. There was a single ENND in the no-IVIG group, which occurred following an IUT at 25 weeks' gestation. Ten neonates required transfusion, 6 in the IVIG group and 4 in the no-IVIG group (Table 3). The Hb levels of the 4 transfused neonates in the IVIG group were 6.3, 8.1, 9.3 and $10.3 \mathrm{~g} / \mathrm{dL}$, respectively. Hb levels for 2 neonates in the IVIG group were not available. The Hb levels of the 4 neonates that were transfused in the no-IVIG group were 6,8 , 10 and $11.5 \mathrm{~g} / \mathrm{dL}$, respectively. Four women had IUTs: 2 in the IVIG group and 2 in the no-IVIG group (Table 4). Patient 2 and patient 4 required a repeat transfusion (Table 4 ). The fetus in the IVIG group was hydropic and required a transfusion at 25 weeks. At 29 weeks' gestation, the fetus was still hydropic and had an $\mathrm{Hb}$ level of $6 \mathrm{~g} /$ dL. A repeat transfusion was performed, and the fetus was delivered at 30 weeks' gestation. The fetus in the no-IVIG group presented as a hydrops fetalis and had transfusions at 22, 23, 25 and 27 weeks' gestation. The pre-transfusion $\mathrm{Hb}$ level after three transfusions was $5.9 \mathrm{~g} / \mathrm{dL}$, and the fetus was still hydropic at the time of the fourth transfusion. This fetus was delivered at 28 weeks' gestation. Among all RhD-sensitised women, 11 had IUFD or ENND in their previous pregnancy. Among the women who received IVIG, 8 had a previous ENND and 2 had had a previous IUFD. Nine of these 10 women had a successful outcome in the index pregnancy in which they had received IVIG. One woman in the IVIG group had an unexplained IUFD. This woman had regular follow-ups, her antibody titre levels remained static and the MCA maximum velocity $\left(\mathrm{V}_{\max }\right)$ did not show any evidence of fetal anaemia. There was a single woman with a previous IUFD in the no-IVIG group. This woman would have been a candidate to receive IVIG. However, she presented at 20 weeks' gestation with a hydropic fetus and opted to have a termination of pregnancy.

\section{Discussion}

In our cohort of $\mathrm{RhD}$-sensitised women, the majority of women who received IVIG had a history of previous IUFD or ENND compared with women who did not receive IVIG (10 v. 1, $p<0.01$; Table 2$)$. This may be explained by the criteria used to administer maternal IVIG. The literature pertaining to the criteria for the use of IVIG in RhDsensitised women is limited. The largest such study was by Zwiers et al. ${ }^{[6]}$ in 2018 and described the outcomes of $24 \mathrm{RhD}$-sensitised women who received IVIG compared with $28 \mathrm{Rh}$-sensitised women who did not. The criteria used by Zwiers et al. ${ }^{[6]}$ for maternal IVIG administration were previous IUFD or ENND related to Rh disease, previous IUT prior to 24 weeks' gestation and women with high maternal antibody titre levels, although the exact titre levels were poorly defined. ${ }^{[6]}$ In our study, the criteria used to administer IVIG were similar to those used by Zwiers et al. ${ }^{[6]}$ However, in addition, our study included women with increasing maternal antibody titres. A maternal antibody titre level $\geq 1: 128$ has been found to correlate with a clinically significant risk of developing fetal anaemia. ${ }^{[3]}$ In our cohort, $64 \%$ of women in the IVIG group and none in the no-IVIG group had an initial maternal antibody titre level $\geq 1: 128(p=0.01)$. There was a statistically significant proportion of women in the IVIG group that had a decrease in antibody titres when compared with those in the no IVIG group ( $43 \%$ v. $11 \%$; $p=0.04$ ). This supports the previously proposed mechanism of action of IVIG in reducing maternal Rh antibody titre production. ${ }^{[5]}$ There were two women in the IVIG group who had an increase in antibody titre levels. Both commenced treatment after 24 weeks' gestation owing to late initiation of antenatal care and delay in approval of treatment by their health insurance. This may suggest that the initiation of IVIG after 20 weeks' gestation may not be as beneficial in reducing maternal antibody titre levels as initiation of IVIG prior to 20 weeks' gestation. There is no literature to support or refute this finding. There were no cases of ENND in the IVIG group and there was a single ENND in the no-IVIG group following an IUT and subsequent preterm labour at 25 weeks' gestation. While comparative statistics cannot be applied here, this death highlights the difficulties of transfusing such a small anaemic fetus. IUT is technically challenging prior to 20 weeks' gestation owing to the small diameter of the umbilical cord. Lindenburg et al. ${ }^{[7]}$

Table 4. Description of the four pregnancies where IUT was performed

\begin{tabular}{|c|c|c|c|c|}
\hline \multirow[b]{2}{*}{ Characteristic } & \multicolumn{2}{|c|}{ IVIG } & \multicolumn{2}{|c|}{ No-IVIG } \\
\hline & P 1 & P 2 & P 3 & P 4 \\
\hline GA at first IUT (days) & 223 & 179 & 180 & 156 \\
\hline Repeat IUT, $n$ & 0 & 3 & 0 & 4 \\
\hline GA at delivery (days) & 224 & 210 & 189 & 196 \\
\hline Birthweight (g) & 1500 & 1610 & 500 & 1100 \\
\hline $\begin{array}{l}\text { Neonatal transfusion } \\
\text { performed }\end{array}$ & Yes & Yes & No & Yes \\
\hline Early neonatal death & No & No & Yes & No \\
\hline
\end{tabular}

Table 3. Obstetric and neonatal outcomes of $\mathrm{RhD}$-sensitised women by treatment group

\begin{tabular}{lll} 
Table 3. Obstetric and neonatal outcomes of RhD-sensitised women by treatment group & No-IVIG $(\boldsymbol{n}=\mathbf{2 8}), \boldsymbol{n}(\%)^{*}$ \\
\hline Outcome & IVIG $(\boldsymbol{n}=\mathbf{1 4}), \boldsymbol{n}(\mathbf{\%})^{*}$ & $26(92)$ \\
\hline Obstetric & $13(92)$ & $2(7)$ \\
Born alive & $1(7)$ & $37(20-39)$ \\
IUFD & $33(31-37)$ & $2990(500-3900)$ \\
GA at delivery (weeks), median (range) & $2180(900-3100)$ & 1.0 \\
Birthweight (g), median (range) & & $4(3.5)$ \\
Neonatal & 0 & $8(14)$ \\
ENND & $6(43)$ & 0.01 \\
Neonatal transfusion & $5(38)$ & 0.01 \\
Phototherapy & $4(29)$ & $6(21)$ \\
Neonatal IVIG & $8(57)$ & 0.47 \\
NICU admission & 0.04 \\
RhD $=$ Rhesus D; IVIG $=$ intravenous immunoglobulin; IUFD $=$ intrauterine fetal death; GA = gestational age; ENND= early neonatal death; NICU = neonatal intensive care unit. & 0.64
\end{tabular}


reported a four-fold increased risk of fetal loss following IUT at $<20$ weeks compared with transfusion after 20 weeks. At later gestational ages, this invasive procedure can be associated with complications such as fetal distress, pre-labour rupture of membranes and preterm delivery. ${ }^{[8]}$ Two fetuses were transfused in each group - one in the IVIG group and one in the no-IVIG group required repeat transfusion (Table 4). The fetus in the IVIG group was hydropic and required a transfusion at 25 weeks' gestation. At 29 weeks' gestation, this fetus remained hydropic, had a repeat transfusion and was delivered at 30 weeks' gestation. The fetus in the no-IVIG group presented as a hydrops fetalis, and received transfusions at 22, 23, 25 and 27 weeks' gestation. In view of the fact that the fetus was still hydropic after 3 IUTs and had an $\mathrm{Hb}$ level of $5.9 \mathrm{~g} / \mathrm{dL}$, the fetus was transfused and subsequently delivered at 28 weeks' gestation. There are two reasons to justify the individualisation of care. The first is the increased risk of fetal demise with repeated transfusions. ${ }^{[8]}$ The second is that the MCA peak systolic velocity is less reliable following multiple intrauterine transfusions. ${ }^{[9]}$ The increase in MCA peak systolic velocity in an anaemic fetus is a reflection of a hyperdynamic fetal circulation due to reduced blood viscosity. When an anaemic fetus is transfused with adult blood, which has an even lower viscosity, the MCA peak systolic velocity may falsely predict anaemia. ${ }^{[9]}$ Zwiers et al. ${ }^{[6]}$ also demonstrated that in pregnancies treated with IVIG, fetal anaemia developed later in gestation, and there were also fewer cases of hydrops fetalis. ${ }^{[6]}$ An Rh-sensitised fetus may require multiple IUTs, and it is important that these IUTs are performed at a dedicated fetal medicine centre to optimise fetal outcomes and to maintain operator skill of cordocentesis and fetal blood transfusion. In our study, the median gestational age at delivery was 33 weeks in the IVIG group and 37 weeks in the untreated group $(p=0.01)$. The decision for delivery in the IVIG group was based on either an increasing maternal antibody titre level or a fetal MCA $\mathrm{V}_{\max }$ indicating the presence of fetal anaemia in a fetus at a viable gestation. Our unit favours delivery once fetal viability has been attained, as there are adequate neonatal facilities in our setting. Other units may opt to continue monitoring an Rh-sensitised woman and to plan delivery at term. However, this expectant approach also carries a risk of adverse fetal and neonatal outcomes. Infants in the IVIG group had a median birthweight of $2180 \mathrm{~g}$ compared with $2990 \mathrm{~g}$ in the no-IVIG group $(p=0.01)$. Prematurity and consequently low birthweight were iatrogenic and attributed to the severity of $\mathrm{RhD}$ sensitisation, necessitating delivery and not the IVIG treatment itself. While overall survival did not differ significantly between the two groups, the neonatal ICU admission, blood transfusion and neonatal IVIG administration occurred more frequently in the IVIG group than the no-IVIG group. This may be explained by iatrogenic prematurity complicated by severe $\mathrm{Rh}$ sensitisation. Ten women in the IVIG group had previously had IUFD or ENND related to Rh disease. Nine of these 10 women had a successful outcome in the index pregnancy in which they received IVIG. This suggests that maternal IVIG may improve pregnancy outcomes in Rh-sensitised women with previous adverse outcomes related to $\mathrm{Rh}$ disease. The findings of the present study are similar to the only prospective study performed in 1991 by Margulies et al..$^{[10]}$ where maternal IVIG was administered to 24 severely Rh-sensitised women. This study found that IVIG reduced maternal antibody titres, decreased fetal haemolysis and delayed IUT if initiated prior to 28 weeks' gestation. ${ }^{[10]}$ However, it is possible that administration of IVIG earlier in gestation is required to reduce $\mathrm{RhD}$ sensitisation and decrease perinatal mortality. Adverse effects of IVIG can be classified as common $(20-40 \%)$, less common $(<5 \%)$ and rare $(<0.1 \%){ }^{[11]}$ Fortunately, common reactions are usually mild, less common reactions are moderate in severity and severe reactions rarely occur. ${ }^{[1]}$ Previous studies on maternal IVIG in Rh disease did not evaluate the maternal effects of this treatment. Among women who received IVIG in our study, there were no adverse maternal effects reported. This suggests that maternal IVIG administration for fetal benefit may not pose a significant risk to maternal health, but this finding would require validation by a much larger and preferably randomised controlled trial. The findings of this retrospective study are limited by the sample size and absence of $\mathrm{Hb}$ levels in a small proportion of neonates. Rh sensitisation in pregnancy is rare owing to the fact that anti-D immunoglobulin is readily available.

\section{Conclusion}

This is the first study in SA to assess the pregnancy outcomes of women receiving IVIG for the treatment of red cell sensitisation. All Rh-sensitised women should be closely monitored and managed at a dedicated fetal medicine centre. The findings of this study suggest that the use of maternal IVIG may provide a successful pregnancy outcome in $\mathrm{RhD}$-sensitised women with previous adverse pregnancy outcomes related to $\mathrm{Rh}$ disease, or women with raised or increasing maternal antibody titre levels who present in the first or early second trimester. Such a strategy allows the option of minimal intervention and IUT as a salvage procedure if severe fetal anaemia develops at a non-viable gestation. In order to advance the quality of research within the public health sector, it is important for institutions to systematically record and retain data.

\section{Declaration. None.}

Acknowledgments. We would like to thank Sister Sharron Bister for her invaluable assistance with the collection of data.

Author contributions. Equal contributions.

Funding. None.

Conflicts of interest. None.

1. Oepkes D. The modern management of red cell alloimmunization. TOG 2003;5(1):15-20. https:// doi.org/10.1576/toag.5.1.15

2. Yang $\mathrm{H}$, Llewellyn $\mathrm{A}$, Walker $\mathrm{R}$, et al. High-throughput, non-invasive prenatal testing for fetal Rhesus D status in RhD-negative women: A systematic review and meta-analysis. BMC Med 2019;17(1):37. https://doi.org/10.1186/s12916-019-1254-4

3. Resnik R, Creasy R, Iams J, Lockwood C, Moore T, Greene M. Haemolytic disease of the fetus and newborn. In: Creasy R, Resniks R, eds. Maternal-Fetal Medicine: Principles and Practice. 7th ed. Philadelphia: Elsevier Saunders, 2014:558-568.

4. Mari G, Deter RL, Carpenter RL. Noninvasive diagnosis by Doppler ultrasonography of fetal Mari G, Deter RL, Carpenter RL. Noninvasive diagnosis by Doppler ultrasonography of fetal
anemia due to maternal red-cell alloimmunization. N Engl J Med 2000;342(1):9-14. https://doi. anemia due to maternal red-cell alloim
org/10.1056/NEJM200001063420102

5. Durandy A, Kaveri SV, Kuipers TW, et al. Intravenous immunoglobulins - understanding properties and mechanisms. Clin Exp Immunol 2009;158(s1):2-13. https://doi.org/10.1111/j.13652249.2009.04022.x

6. Zwiers C, Van De Bom JG, Van Kemp IL, et al. Postponing early intrauterine transfusion with fetus and newborn. Am J Obstet Gynaecol 2018;219(3):291.e1-291.e9. https://doi.org/10.1016/j. ajog.2018.06.007

7. Lindenburg IT, Van Kemp IL, Van Zwet EW. Increased perinatal loss after intrauterine transfusion Lindenburg IT, Van Kemp IL, Van Zwet EW. Increased perinatal loss after intrauterine transfusion
for alloimmune anaemia before 20 weeks of gestation. BJOG 2013;120(7):847-852. https://doi. for alloimmune anaemia before
org/10.1111/1471-0528.12063

8. Zwiers C, Lindenburg I, Klumper F, De Haas M, Oepkes D, Van Kemp I. Complications of intrauterine intravascular blood transfusion: Lessons learned after 1678 procedures. Ultrasound Obstet Gynaecol 2017;50(2):180-186. https://doi.org/10.1002/uog.17319.

9. Scheier M, Hernandez-Andrade E, Fonseca EB, Nicolaides KH. Prediction of severe fetal anemia in red blood cell alloimmunization after previous intrauterine transfusions. Am J Obstet Gynaecol 2006,195(6):1550-1556. https://doi.org/10.1016/j.ajog.2006.03.060

10. Margulies M, Voto LS, Mathet E. High-dose intravenous IgG for the treatment of severe Rhesus alloimmunization. Vox Sang 1991;61(3):181-189. https://doi.org/10.1111/j.1423-0410.1991.tb00944.x

11. Peter J, Heckmann J, Norvitsky N. Recommendations for the use of immunoglobulin therapy for immunomodulation and antibody replacement. S Afr Med J 2014;104(11):7306. https://doi. org/10.7196/samj. 8965 